\title{
Reflexiones sobre la dimensión transnacional de la literatura contemporánea en Italia y en Europa
}

\author{
Franca Sinopoli \\ «La Sapienza»ç Università di Roma \\ Traducción del italiano: \\ Valeria Ansó - Marco Franzoso • • \\ Universidad Nacional del Litoral
}

\section{Resumen}

El objetivo de este artículo es reflejar la dimensión transnacional de muchos autores contemporáneos en Italia y dentro del marco del contexto europeo. El transnacionalismo asigna un rol fundamental a la construcción de una imaginación transfronteriza o híbrida, en diferentes niveles culturales y comunicacionales, por ejemplo alentando la atención a aquellas producciones en campos literarios, cinematográfico, artísticos que serían impensables si confiamos en el concepto tradicional de espacio donde la geografía física, cultural, política y social coinciden perfectamente. El paradigma del transnacionalismo también echa luz a las producciones de autores literarios contemporáneos que tienen backgrounds multiculturales y multilingüísticos, viven y operan en un contexto nacional específico o, en diferentes períodos, se mueven de una nación a otra, o incluso siguen trayectorias intercontinentales.

\section{Palabras clave}

- Transnacionalismo · Literatura contemporánea · Italia · Europa · Translingüismo

\footnotetext{
- Franca Sinopoli es Profesora Asociada de Crítica Literaria y Literaturas Comparadas en los cursos de estudio trienales y magistrales de Letras de «La Sapienza» Università di Roma. Sus campos de investigación son: la metodología del estudio comparado de la literatura, la historiografía y la crítica literaria europea de base comparatista del siglo XVIII hasta hoy, las formas de transnacionalismo literario en la modernidad (estudios sobre traducción, diáspora, translingüismo literario). Es titular de un proyecto de investigación de Ateneo (2019-2022) sobre «Narrating the trauma in european literatures and cultures from the second half of the 19 th century to the "late modernity": a comparative approach to memory and post-memory narratives in Italy and Europe». Sitio web institucional: https://www.lettere.uniroma1.it/users/franca-sinopoli - - Valeria Ansó es Profesora y Licenciada en Letras por la Universidad Nacional del Litoral y la Università Ca'Foscari de Venecia. Es Doctoranda en Humanidades con orientación en Letras en UNL. Es profesora en diversas cátedras de literaturas europeas traducidas en UNL y la Universidad Autónoma de Entre Ríos. Marco Franzoso es graduado en «Lingue e letterature europee, americane e postcoloniali» por la Università Ca'Foscari de Venecia y es Licenciado en Letras por la Universidad Nacional del Litoral. Doctorando en Humanidades con orientación en Letras en UNL y profesor de la cátedra de Italiano de la misma institución.
} 


\section{Abstract}

This article aims to reflect is to reflect on the transnational dimension of many contemporary authors in Italy and within the framework of the European context. Transnationalism assigns a pivotal role to the construction of a cross-border or hybrid imagination, on different cultural and communicational levels, for example by encouraging the attention to those productions in the literary, cinematic, and artistic field which would be unthinkable if we relied on the traditional concept of space where physical, cultural, political and social geography are perfectly matched. The paradigm of transnationalism also sheds light on the productions of contemporary literary authors who have multicultural and multilingual backgrounds, live and operate in a specific national context or in different periods, move from one nation to another, or even follow intercontinental trajectories.

\section{Keywords}

Transnationalism · Contemporary literature · Italy · Europe . Translingualism

Como fue observado por la crítica académica oltreoceanica, que especialmente en los EEUU elaboró hace tiempo una profunda reflexión sobre los límites de la idea de literatura nacional, el cambio de Italia de tierra de emigración — como fue hasta la segunda posguerra - a tierra de inmigración —en la que se convirtió en los últimos treinta años- determinó una recuperación de territorios de la escritura literaria que tradicionalmente se encontraban al margen del concepto de "literatura» y que por ende fueron dejados de lado de los estudios e investigaciones universitarias. Me refiero en particular no solo a los intereses crecientes en Italia por el área de los llamados «Italian American Studies» (IAS) ${ }^{1}$, nacidos en las universidades norteamericanas y luego recientemente legitimados también por nosotros a nivel académico por los estudios de la americanística y la italianística ${ }^{2}$, sino a la posibilidad de dar espacio en nuestra propia italianística a un concepto transnacional de literatura. Este llega, por un lado, a producir un interés hacia obras literarias originadas en el siglo XX por los procesos migratorios desde/hacia Italia y por otro a derivar una puesta en crisis del paradigma de oposición y dualidad centro-periferia o canon-frontera, especialmente para lo relativo al siglo XX, paradigma de lectura tan penetrante en los estudios literarios como para ser sobreentendido ${ }^{3}$. 
Es evidente la problematicidad que este cambio de perspectiva comporta y no menos importante la cuestión de cómo individuar el significado que reviste para la cultura italiana del siglo XX un patrimonio literario expresado en italiano o en otras lenguas por sujetos emigrados de Italia y que sin embargo ha dicho y dice todavía mucho sobre la idea de italianidad de proveniencia. También, por otra parte, la cuestión de cómo hacer ingresar al canon literario contemporáneo obras de autores de origen extranjero presentes en Italia pero que usan el italiano como lengua de elección. Tales escrituras no encuentran necesariamente fácil ubicación en «el canon» en tanto, con su pasado lingüístico y cultural diferente, agrietan cualquier acepción radicada y uniforme de "lo canónico» vigente en el ámbito nacional ${ }^{4}$.

Un signo ulterior del cambio en la percepción de este tipo de estudios es la reciente revitalización de congresos dedicados, en Italia ${ }^{5}$ y en el extranjero ${ }^{6}$, a la recodificación de las mismas categorías utilizadas para afrontar la vasta fenomenología literaria y cultural en general relacionada con la emigración y/o inmigración italiana. Conceptos como movilidad, diáspora, migración, transnacionalismo se revelan útiles para releer en el plano de la producción cultural no solos las viejas y nuevas experiencias migratorias, sino también para reconfigurar el corpus literario resultante en términos de pertenencias múltiples a diferentes contextos lingüísticoculturales. Se superan así las clasificaciones historiográficas que se remontan más o menos explícitamente al paradigma centro- periferia, basado en aquella que la historiadora italoamericana Donna R. Gabaccia circunscribe como expresión de una tiranía de lo nacional ${ }^{7}$. En tal contexto resulta particularmente iluminante, en el campo sociológico global (global sociology), que

los migrantes transnacionales sean portadores de divided loyalties, dobles ciudadanías o nacionalidades que ahora una mitad de los estados del mundo de alguna manera admite y reconoce, y como ellos ejercitan un soft power de hibridación a través de la constitución de estilos de vida e instituciones locales que toman ya sea de la sociedad originaria como de la nueva patria ${ }^{8}$.

Quisiera tocar un aspecto de la cuestión general apenas delineada con la intención no de agotarla sino de ofrecerla a las reflexiones de la comunidad de estudiosos interesados en el uso del concepto de transnacionalismo en los estudios literarios sobre Italia.

Algunas intervenciones teóricas elaboradas en los últimos años en el campo del comparatismo literario europeo orientado al estudio del transnacionalismo cultural y literario ${ }^{9}$ propusieron interesantes reflexiones sobre políticas de inclusión y exclusión. Estas políticas están activas en la construcción y en la transmisión de un canon literario (ya sea a nivel nacional como continental europeo), proporcionando en particular disparadores interesantes para razonar en torno a los significados del término «transnacional» y del de "canon» a propósito de las producciones literarias contemporáneas de parte de autores y autoras que tengan un background multicultural y plurilingüe - aun viviendo y operando en uno de los contextos nacionales europeos o también en más de uno, en el tiempo, en tanto reubicados en una a otra nación de Europa.

En el campo sociológico, Steven Vertovec en la introducción a su volumen de 2009 Transnacionalism, después de haber descrito constantes y variantes del migrant trasnationalism y después de haber afrontado las principales objeciones críticas al uso del concepto mismo de transnacionalismo, sugiere la necesidad de tomar en consideración los espacios de la actividad humana aún inexplorados desde una 
perspectiva transnacional. Existen diversos niveles de actividad transnacional que exigen ser individuados y analizados. La perspectiva esencialmente sociológica de Vertovec no comprende fenómenos culturales de tipo estético, como la literatura, aunque tal apertura induce a interrogarse sobre los efectos del transnacionalismo de los migrantes en este ámbito específico. Pero primero es útil recapitular algunos elementos típicos del transnacionalismo de los migrantes contemporáneos según Vertovec, gracias a los cuales estos se diferencian de las formas precedentes de transnacionalismo. Cuatro en particular me parecen útiles para la comprensión de las consecuencias del fenómeno sobre el plano cultural: a) efectos de la velocidad de las tecnologías de la comunicación sobre la calidad de las relaciones del migrante no solo con el propio contexto de origen sino en general con el mundo; b) el contexto de proveniencia es, a su vez, influenciado por los eventos y los valores que viven y expresan sus connacionales en el extranjero, o sea que habría una suerte de circularidad o cuanto menos una interacción entre aquellos que migraron y aquellos que permanecieron en el lugar de origen; c) la propuesta de introducir la doble ciudadanía para aquellos que deseen naturalizarse en el país de acogida; d) el coming out de la propia identidad transnacional gracias a más de veinte años de políticas antirracistas y de batallas por los derechos civiles que tuvieron lugar en muchos países occidentales (Vertovec, 2009:14-16).

La superación del sentido de no-pertenencia del sujeto transnacional es posible, entonces, por la difusión de una mentalidad dispuesta a concebir la existencia de subjetividades humanas manifiestamente no radicadas o ya no radicadas en una sola lengua, cultura, milieu nacional, etc. Una de las consecuencias positivas de este cambio de mentalidad sería por ejemplo la gran oportunidad de ver de otra manera que ese sentido de no-pertenencia expresado por los sujetos transnacionales no tiene una consistencia de tipo esencialista o natural, sino que es el fruto de un artificio prospectivo que atribuye valor de verdad únicamente al paradigma monocultural y monolingüístico sobre el que se funda el concepto moderno dieciochesco de nación. Algunos recientes volúmenes aparecidos en el ámbito comparatista, entre los cuales los citados previamente, publicados respectivamente por Theo D'haen y Mads Rosenthal Thomsen, nos permite reubicar el discurso de Vertovec en el plano de los estudios literarios y profundizar el significado del uso de la categoría de «transnacionalismo» en la reflexión crítica sobre la literatura contemporánea, y en particular sobre la europea. Solo un análisis de las múltiples acepciones y modalidades de uso de tal categoría en el discurso crítico contemporáneo podrá conducir luego, en un segundo momento, al tentativo de dar una representación general y verosímil de la transnacionalidad europea en la literatura a partir de la segunda mitad del siglo XX. Ambos estudios, después de haber recorrido la historia semántica de la «literatura mundial», los diversos paradigmas de la categoría de mundialidad literaria y sus relaciones con el estudio del canon de la literatura en ámbito comparatista, recontextualizan cada uno a su modo tal categoría de «literatura mundial» en la contemporaneidad. En particular, ambos volúmenes asignan una cierta importancia a la literatura producida en las lenguas europeas de autores de nacionalidad y cultura no europeas, pero lo hacen siguiendo dos pistas diferentes: D'haen lo hace introduciendo la cuestión del "poscolonial» y de las «alofonías» (francofonía, in primis) como producto de occidente (D'haen, 2012) ${ }^{10}$ y por tanto no realmente representativas ni de los autores migrantes ni de las poblaciones africanas y asiáticas, en su mayoría no diaspóricas; mientras Rosendhal Thomsen se concentra directamente 
en la «migrant literature» circunscribiéndola como expresión actual de la literatura transnacional. A propósito de la publicación del manifiesto Pour une littératuremonde en français en Le Monde (16 de marzo de 2007) por parte de 44 autores de la llamada francofonía, D'haen recuerda justamente que en ámbito comparatista en 2006 Amily Apter ya había identificado una posibilidad del desvinculación de la francofonía al enlace colonial con Francia a través del enfoque de la dimensión transnacional de la lengua francesa en las llamadas «linguistic contact zones all over the world in which French [...] is one of many languages in play» (Apter, 2006: 55). Se trata, prácticamente, de considerar nuevas posibilidades de significado del término transnacional, más allá del vínculo entre excolonias y naciones europeas.

Del mismo modo, la búsqueda de «nuevos cánones» — como recientemente se propone en Italia en el área de los estudios de anglicística ${ }^{11}$, pero cuyos resultados en el plano crítico y teórico general pueden ser útilmente importados a otros contextos disciplinares - es sumamente relevante para rediseñar un cuadro de representaciones más articulado de la contemporaneidad literaria en Europa, y por lo tanto también en Italia. Esto evitaría reducir la literatura transnacional a un subsistema de la literatura nacional y viceversa, tomando en consideración la posibilidad de prefigurar mediante un acercamiento comparatista una idea de literatura nacional atravesada también por una dimensión transnacional que se concretiza en los productos literarios derivados de las numerosas historias de despatrios y diásporas desde/hacia Italia (Martelli, 2009: 725-764).

Además del cuadro de referencias críticas y teóricas recién mencionadas, quisiera mencionar brevemente un proyecto de investigación sobre la dimensión transnacional de la literatura europea contemporánea, iniciado hace algunos años, junto a los colegas Fridrun Rinner (Université de Provence), Helga Mitterbauer (Université Libre de Bruxelles), Myriam Geiser (Université Stendhal - Grenoble), Jeanne Glesener (Université du Luxembourg) y Sandra Vlasta (Österreichische Akademie des Wissenschaften) ${ }^{12}$. Se trata de un proyecto de investigación plurianual y realizado con base internacional con el patrocinio de la International Comparative Literatura Association/ Association Internationale de Litérature Compareé, cuyos resultados se publicarán en un volumen que se encuentra en preparación por la editorial Benjamins. Nuestra investigación titulada «Transculture: Migration and Literature in contemporary Europe», que tiene una perspectiva europea dirigida a la producción literaria transnacional de la segunda mitad del siglo XX, ha tenido no obstante que considerar el aspecto determinante constituido por las diversas políticas culturales nacionales, que parecen un factor del cual no se puede prescindir en tanto es útil para explicar la heterogeneidad en la recepción de estos autores en su ubicación respecto a la cuestión del canon literario contemporáneo. Todo ello a la luz de cambios sociales y culturales ocurridos en Europa como resultado de diversas corrientes migratorias que cruzaron el continente en los últimos decenios del siglo XX, y que continúan impactando. El concepto de «transnacionalidad» y la renegociación de "nuevos cánones» en la literatura contemporánea parecen ser los dos lugares del discurso crítico en los cuales mayormente confluyen las reflexiones sobre las diversas experiencias literarias a las cuales nuestra investigación se refiere. Ambos son fuertemente deudores del contexto político-cultural o más bien condicionados por la fuerza - a veces emancipadora o viceversa - de su acción homologadora, que acepta con mucha dificultad, y de acuerdo con algunos compromisos, la inclusión de autores transnacionales en el modelo de canon nacional prevalente. 
La transnacionalidad literaria es, de hecho, un campo complejo de relaciones que no es posible limitar a la condición postcolonial, o al menos a su acepción nacionalista y monolingüista que privilegia, de hecho, la relación bilateral entre algunas naciones europeas y sus excolonias. Esto es evidente si hablamos de transnacionalismo a escala europea, en modo particular en el caso de Italia, donde el mayor número de escritores y escritoras de origen extranjero que utilizan el italiano como lengua de expresión literaria provienen de países que no tienen ninguna relación directa con el pasado colonial de nuestra nación. Uno de los nudos críticos a tomar en cuenta es, por lo tanto, la amplia cuestión terminológica mediante la cual es posible discutir las varias modalidades de nombrar tal literatura a partir de diversos conceptos provenientes de las áreas de la alofonía, no exclusivamente y en sentido estrecho definible como postcolonial - a menos que con tal término no entendamos referirnos a una época y a una cultural global, en cuyo ámbito se comparten los efectos a escala mundial de diversos colonialismos históricos. Junto a la presencia de una verdadera pluralidad de nomenclaturas (por ejemplo, haciendo referencia al léxico internacional presente en este ámbito de estudios, el uso y la difusión de términos como ibridity, métissage, creolisation, nomadism, migration, diaspora, cosmopolitanism, exile, displacement, mobility, interculturalism, transculturalism, globalism, etc.) deben ser consideradas las estrategias textuales y las tendencias temáticas, el plurilingüismo, el translingüismo, la autotraducción, las modalidades de recepción y de ubicación de estos textos respecto al canon literario tradicional, el rol de los escritores transnacionales en la cultura contemporánea (por ejemplo, su participación más o menos activa en las políticas culturales del país en el que residen), las traducciones de sus obras en el extranjero, el impacto de estas traducciones en diversos contextos nacionales y en relación a sus cánones institucionalizados, para retomar un disparador interesante ofrecido por la contribución de Siri Nergaard al volumen ya mencionado Altri canoni, altri libri (2011), y así sucesivamente.

Para entrar más en detalle en la cuestión de la "política cultural», esta se podría también parafrasear a través de la pregunta sobre cuáles son las formas de acceso del escritor transnacional al campo literario de la «nación» tal como es delimitado por las instituciones vigentes en un determinado país. En tal caso, una primera diferencia debe ser hecha entre naciones como Italia o Alemania que presentan, limitándonos a las primeras generaciones, una producción diversificada desde el punto de vista del background cultural y lingüístico de los autores, y países como Inglaterra, Espańa o Francia. En estos últimos donde es mayor el porcentaje de autores llamados "postcoloniales» y entonces no deben operar un code-switching una vez que se proponen profesionalmente como autores de la nación de llegada, dado que comparten ya la lengua. Resulta, por ejemplo, que al autor translingüe $e^{13}$ se concede menor libertad en el perfil de la creatividad lingüística por parte de los reguladores del campo literario (desde el copy right a la cooperación en la escritura) con respecto al postcolonial, del que - especialmente si es de segunda generación - se podría esperar operatividad interlingüística entre la lengua colonial y otras lenguas presentes en su bagaje cultural familiar. Pero también en este caso es difícil generalizar, teniendo en cuenta el hecho de que, en el caso de Italia, la permanencia de la lengua colonial en los territorios ocupados principalmente en África oriental fue discontinua, no sistemática e intrusiva desde el punto de vista de la penetración intergeneracional, a causa de la breve duración no tanto del colonialismo italiano en sí — como tanto nos enseñaron los historiadores de los 
años 70 del siglo pasado en adelante—, sino de la institución del imperio colonial en cuanto tal, con la mala y discriminadora política lingüístico- cultural adoptada en las colonias. Basta pensar, como prueba de lo dicho, en la actual exigüidad del corpus estrictamente postcolonial de autores que escriben en italiano, esto es de sujetos cuyo origen familiar interesa a las ex colonias italianas (autores y autoras de primera y segunda generación). Para delinear un campo de indagación lo suficientemente consistente y dominable como "postcolonial» fue necesario importar del interior de tal perspectiva autores italianos que trataron narrativamente el periodo colonial por experiencia directa ${ }^{14}$ (Flaiano, Tobino) o indirecta (Pascoli, D'Annunzio, Maria Luisa Astaldi) o reconstruida a posteriori (Longo, Lucarlli, Domenichelli) o autores de origen italiano que nacieron y vivieron en las ex colonias (Erminia dell'Oro), junto a escritores de origen eritreo, somalí y etíope de primera o segunda generación ${ }^{15}$.

Todo esto para ejemplificar la dificultad de generalizar cuando nos movemos en una perspectiva europea y se busca diferenciar el nivel de libertad en el plano de la creatividad lingüística y literaria que se reconoce a los autores transnacionales de los respectivos campos literarios nacionales en los que ellos operan, desde el momento en que etiquetas como «literatura de migración» $y$ «literatura postcolonial» no cubren exactamente el mismo género de autores cuando se pasa de un contexto nacional a otro. Una política cultural determina también el peso específico que el autor transnacional puede adquirir en la «representación» de la literatura nacional vehiculada por las instituciones educativas y los medios de comunicación de masa (TV e internet). En este caso, sea en lo que respecta a la repercusión en el plano colectivo de los contenidos massmediáticos creados con objetivos educativos, como en lo concerniente al espacio tradicionalmente dedicado en los diarios y la TV al mercado editorial relativo a tal tipología de autores. Numerosos disparadores en esa dirección se pueden encontrar en el documento sobre la política cultural europea dedicado a "Cultura e settore audiovisivo. Celebrare la diversità culturale dell'Europa» publicado por la Comisión Europea en junio de 2013, entre cuyas prioridades se lee la promoción de la diversidad cultural y el diálogo intercultural, con la institución ya desde el 2009 de un premio europeo específicamente dedicado a la literatura, en el que participan los autores de los países que participan del programa cultural de la UE «Europa creativa» (adhieren también algunos países extra-UE). El premio de la Unión Europea para la literatura, gestionado por un consorcio formado por las asociaciones europeas de escritores, libreros y editores, está de hecho dirigido actualmente a 44 países de toda Europa, entre los cuales se encuentran los 28 países miembros. Cada año los jurados nacionales de un tercio de los países proclaman los vencedores, de modo que todos los países sean representados en el arco de un trienio. Esta oportunidad, por ejemplo, podría ser tomada para superar, especialmente en el caso de Italia, la tradición de los premios-ghetto dedicados a autores de origen no italiano y similares, iniciada en los ańos noventa, introyectando el principio de la representatividad a nivel europeo de nuestros autores translingües en cuanto autores italianos. Desde el momento en que el premio está dedicado a «to promote cross-border mobility of those working in the cultural sector; to encourage the transnational circulation of cultural and artistic output; and to foster intercultural dialogue» ${ }^{16}$ esto podría ser llevado adelante a través de un jurado nacional sensible a los cambios y a las diferentes articulaciones del actual panorama literario y cultural italiano, en tanto los can- 
didatos al premio europeo serían propuestos por jurados individuales nacionales. Es interesante también la comparación entre las candidaturas propuestas por los diversos jurados nacionales competidores del premio europeo, que revelan diversas políticas culturales y diversas variaciones de la relación entre poder editorial y cultura, a propósito de las cuales cada uno puede hacerse una idea consultando el dossier del premio disponible online en lengua inglesa y francesa.

Otro aspecto a considerar en lo que respecta a la política cultural está claramente relacionado con por la recepción académica de la literatura transnacional, que en su escala europea muestra un notable desarrollo en los últimos decenios con un consecuente incremento a nivel de estudios y bibliografía específica ${ }^{17}$. También este aspecto es examinado por el ya mencionado proyecto "Transculture», que está trabajando sobre las formas de internacionalización del campo literario y de su reorganización a la luz de la superación del modelo cultural «centro-periferia» $\mathrm{y}$, en consecuencia, del pasaje de modelos de lectura crítica marginadores (como el de dominante sociológica) a modelos de recepción más complejos en cuya comprensión sociopolítica y culturalista se cruzan formas de análisis dirigidas a la dimensión específicamente literaria y estética de los textos. En esta dirección, un giro muy interesante es aquel representado por el estudio de los pasajes de modelos de critica académica de un contexto a otro de Europa, sobre todo entre países contiguos lingüísticamente (como Alemania y Austria, Francia y Bélgica), o culturalmente (como Italia y Francia). En ellos la nación que tiene una recepción más reciente de tal fenómeno literario se apoya en un primer momento en los modelos de análisis crítico y de definición teórica elaborados en un contexto lingüístico y culturalmente próximo, con todos los problemas de malentendido y de condicionamiento ideológico que esta mutación de paradigmas teórico- crítico puede comportar. En síntesis, el cuadro europeo es necesario para estudiar el nexo entre literaturas transnacionales y políticas culturales, así como en el plano de la rediscusión de la idea de "canon" se trabajó mucho en los últimos años y en diversos campos disciplinares para reorientar tal cuestión en sentido histórico, cultural y diferencial. No menos importante es la gran contribución de los estudios sobre la traducción para la puesta en evidencia del condicionamiento de los cánones nacionales, y de las políticas culturales a ellos subyacentes, operantes en casos específicos en contra o viceversa a favor de la recepción de las literatura extranjeras y de un pasaje transnacional y translingüístico del patrimonio literario de las mismas naciones europeas.

\author{
Notas \\ ${ }^{1}$ Cfr. Pietralunga, M. (2010). «Italian American Studies in Italy». Véase \\ también: Krase, J. (ed.) (2011) The Status of Interpretation in Italian \\ American Studies. New York, Forum Italicum Publishing. \\ ${ }^{2}$ Válidas y pioneras excepciones las constituyen en Italia, por ejemplo, en \\ el ámbito de la italianística, los estudios sobre literatura italoamericana de \\ Francisco Durante, Emilio Franzina, Margarita Ganeri, Martino Marazzi, \\ Sebastiano Martelli, Caterina Romeo. Para estudios análogos sobre la
}


literatura de la migración italiana en otros contextos culturales (desde el resto del continente americano a Australia, África, Asia y Europa) se debe partir del también pionero volumen colectivo dirigido por JeanJacques Marchand (1991) La letteratura dell'emigrazione. Gli scrittori di lingua italiana nel mondo.

${ }^{3}$ El adjetivo «transnacional» es utilizado en el volumen Shifting and Shaping a National Identity: Transnational Writers and Pluriculturalism in Italy Today, ed. por Russo Bullaro, G. y Benelli, E. (2014). Véanse también mis trabajos de 2012 y 2014.

${ }^{4}$ El estudio de la literatura llamada «de migración» en Italia presenta en el estado actual una bibliografía sólida y articulada en ensayos y monografías. Me limito a mencionar aquí solo las principales monografías académicas a esta dedicadas, partiendo de las más recientes: Lecomte (2018), Negro (2015), Moll (2015), Burns (2013), Mengozzi (2013), Lori (2013), Fracassa (2012), Morace (2012), Kiemle (2011), Comberiati (2010), Mauceri e Negro (2009), Parati (2005), Ponzanesi (2004), Gnisci (2003).

${ }^{5}$ No por último quisiera citar el congreso Emigrazione italiana: percorsi interpretativi tra diaspora, transnazionalismo e generazioni realizado en Turín en 2004 y curyos resultados fueron recogidos en el volumen a cargo de Maddalena Tirabassi (2005) Itinera. Paradigmi delle migrazioni italiane. Por lo referido a la producción derivada de la inmigración en Italia, véanse las actas de un congreso realizado en Bolońa, Leggere il testo e il mondo. Vent'anni di scritture della migrazione in Italia, a cura di Fulvio Pezzarossa e Ilaria Rossini (2011).

${ }^{6}$ Véanse por ejemplo los congresos neoyorquinos Migrating in and out of Italy (John D. Calandra Italian American Institute, New York, 25 ${ }^{\text {th }}$ $-26^{\text {th }}$ February 2011) y Lingue migranti: the Global Languages of Italy and the Diaspora (John D. Calandra Italian American Institute, New York, $26^{\text {th }}-27^{\text {th }}$ April 2013). Pero como ejemplo de la compleja dimensión de la migración y de sus numerosas narraciones se puede citar el volumen misceláneo Borderlines. Migrazioni e identità nel Novecento, a cargo de Jennifer Burns e Loredana Polezzi (2003), al cual le sigue idealmente I confini della scrittura. Il dispatrio nei testi letterari, a cargo de Franca Sinopoli e Silvia Tatti, (2005), ambos frutos de dos congresos.

${ }^{7}$ Véase Gabaccia, Donna R., «Diaspore, discipline e migrazioni di massa dall'Italia» en Itinera (Tirabassi, 2005) y su libro Emigranti. Le diaspore degli italiani dal Medioevo (2003).

${ }^{8}$ Cfr. Tirabassi, Maddalena (2005) y de la misma autora con Patrizia Audenino (2008).

${ }^{9}$ Me refiero particularmente a Rosendhal Thomsen, Mads, Mapping World Literature. International Canonization and Transnational Literatures (London, Continuum, 2008) y D'haen, Theo, The Routledge Concise History of World Literature (London and New York, Routledge, 2012)

${ }^{10}$ "Postcolonialism can be seen as a projection of, rather that resistance to, Western thought» (D'haen, 2012:151)

${ }^{11}$ Me refiero al volumen Altri canoni / canoni altri (2011) a cargo de Ornella de Zordo y Fiorenzo Fantaccini, que contiene intervenciones pro- 
venientes de diversas áreas disciplinares. En el ámbito del comparatismo véase el artículo de Mario Domenichelli (2007) «Il canone occidentale anglo-americano e le letterature comparate».

${ }^{12}$ Remito al sitio web oficial de la investigación: https://www.uantwerpen.be/en/projects/chlel/about-chlel/members-and-project-directors/. La bibliografía internacional sobre este campo de estudios es muy vasta, para una selección de la misma remito a mi reciente contribución: Caratteri transnazionali e translinguismo nella letteratura italiana contemporanea, en La Modernità letteraria, vol. 8, 2015, pp. 53-63. A la "transculturalidad» literaria italiana está dedicado, en cambio, el volumen de Martha Kleinhans e Richard Schwaderer (Hrsg.) (2013), Transkulturelle italophone Literatur/Letteratura italofona transculturale. ${ }^{13}$ Para una definición del escritor translingües véase Kellman (2007). ${ }^{14}$ Cfr. Fracassa (2012) y Derobertis, Roberto (2010).

${ }^{15}$ Para un análisis monográfico sistemático de los autores y autoras postcoloniales cuya cultura se origina en parte en los países del Cuerno de África remito al reciente volumen de Maria Grazia Nero (2015), Il mondo, il grido, la parola. La questione linguistica nella letteratura postcoloniale italiana, en particular a los capítulos $2-5$ y a la rica bibliografía final. La estudiosa vuelve sobre la fundamental voluntad de renovar la memoria de lo omitido de la experiencia colonial italiana y en la elección motivada y meditada de escribir en la lengua de los ex colonizadores los factores determinantes que distinguen la escritura postcolonial de la literatura de la migración producida en Italia. Para un tratamiento colectivo de la postcolonialidad italiana se remite en Italia a Lombardi-Diop y Romeo (2014). Otros estudios en parte o totalmente dedicados a la literatura, sobre todo a la narrativa, postcolonial en Italia son de Benvenuti (2012), Comberiati (2012), Lori (2013). Sobre el postcolonial italiano léase también el fascículo doble de Narrativa, núm. 33/34, 2011/12, a cargo de Silvia Contarini, Giuliana Pias, Lucia Quaquarelli. Un perfil de la literatura postcolonial italiana es el del estudioso italosomalí Ali Mumin Ahad, "Corno d'Africa. L'ex-impero italiano» en Gnisci (2006). Así como para la literatura de la migración, la bibliografía relacionada a la literatura postcolonial italiana, publicada en Italia y en el extranjero, aun siendo reciente está en constante crecimiento, principalmente gracias a dos factores de novedad: la ampliación de perspectivas y la innovación metodológica de los estudios sobre la literatura italiana contemporánea, y no menos importante la comparación con situaciones análogas a nivel europeo, que estimularon también en Italia la producción de trabajos sólidos sobre el tema, fruto de investigaciones profundizadas y bien documentadas a nivel internacional.

${ }^{16}$ Véase http://www.euprizeliterature.eu/what-eupl

${ }^{17}$ Se puede partir, por ejemplo, del volumen Migrant Cartographies: New Cultural and New Literary Spaces in Postcolonial Europe, editado por Sandra Ponzanesi y Daniela Merolla (2005) y llegar al reciente Contemporary Developments in Emergent Literatures and the New Europe, editado por César Dominguez y Manus O’Dwyer (2014). 
Referencias bibliográficas

Ahad, A.M. (2006). Corno d'Africa. L'ex-impero italiano. En Gnisci (ed.), Nuovo Planetario Italiano (pp. 241-293). Edizioni Città Aperta. Apter, E. (2006). "Je ne crois pas beaucoup à la littérature comparée». Universal Poetics and Postcolonial Comparatism. En Saussy, H. (ed) Comparative Literature in an Age of Globalization. The Johns Hopkins University Press.

Benvenuti, G. (2012). Romanzo neostorico italiano. Carocci.

Burns, J. (2013). Migrant Imaginaries Figures in Italian Migration Literature. Peter Lang.

Burns, J. y Polezzi, L. (2003). Borderlines. Migrazioni e identità nel Novecento. Cosmo Iannone.

Comberiati, D. (2010). Scrivere nella lingua dell'altro. La letteratura degli immigrati in Italia (1989-2007). Peter Lang.

Comberiati, D. (2012). Ecrire hors du centre: exemples d'écrivains italophones: la littérature italienne contemporaine entre migration et postcolonialité. Editions Universitaires Européennes.

De Zordo, O. y Fantaccini, F. (2011). Altri canoni / canoni altri. University Press

Derobertis, R. (dir.) (2010). Fuori centro. Percorsi postcoloniali nella letteratura italiana. Aracne.

D'Haen, T. (2012). The Routledge Concise History of World Literature. Routledge.

Domenichelli, M. (2007). Il canone occidentale anglo-americano e le letterature comparate. En Balestra, G. e Mochi, G., Ripensare il canone. La letteratura inglese e angloamericana (pp. 185-196). Artemide Dominguez, C. y O’Dwyer, M. (ed.) (2014). Contemporary Developments in Emergent Literatures and the New Europe. USC Editora clave.

Fracassa, U. (2012). Patria e lettere. Per una critica della letteratura postcoloniale e migrante in Italia. Perrone.

Gabaccia, D. (2003). Emigranti. Le diaspore degli italiani dal Medioevo. Einaudi.

Gnisci, A. (2003). Creolizzare l'Europa. Letteratura e migrazione. Meltemi. Kellman, S. (2007). Scrivere tra le lingue. Edizione Città aperta.

KIEmLe, C. (2011). Ways out of Babel: Linguistic and Cultural Diversity in Contemporary Literature in Italy Exploring Multilingualism in the Works of Immigrated Writers. Trier.

Kleinhans, M. y Schwaderer, R. (Ed.) (2013). Transkulturelle italophone Literatur/Letteratura italofona transculturale. Königshausen \& Neumann. Krase, J. (ed.) (2011). The Status of Interpretation in Italian American Studies. Forum Italicum Publishing.

Lecomte, M. (2018). Di un poetico altrove. Poesia transnazionale italofona (1960-2016). Franco Cesati Editore.

Lombardi-Diop, C. y Romeo, C. (Dir.) (2014). L'Italia postcoloniale. Le Monnier.

LORI, L. (2013) Inchiostro d'Africa. La letteratura postcoloniale somala tra diaspora e identità. Ombre corte.

Marchand, J.J. (1991). La letteratura dell'emigrazione. Gli scrittori di lingua italiana nel mondo. Edizioni della Fondazione Giovanni Agnelli 
Martelli, S. (2009). Letteratura delle migrazioni. En Storia d'Italia. Annali, vol. 24: Migrazioni. Einaudi.

Mauceri, C. y Negro, G. (2009). Nuovo immaginario italiano. Sinnos. Mengozzi, C. (2013). Narrazioni contese. Ventanni di scritture italiane della migrazione. Carocci.

Moll, N. (2015). L'infinito sotto casa. Letteratura e transculturalità nell'Italia contemporanea. Pàtron.

Morace, R. (2012). Letteratura-mondo italiana. ETS.

Nero, M.G. (2015). Il mondo, il grido, la parola. La questione linguistica nella letteratura postcoloniale italiana. Franco Cesati Editore.

Parati, G. (2005). Migration Italy. The Art of Talking Back in a Destination Culture. University of Toronto Press.

Pezzarossa, F. y Rossini, R. (dir.) (2011). Leggere il testo e il mondo. Ventanni di scritture della migrazione in Italia. Clueb.

Pietralunga, M. (2010). Italian American Studies in Italy. En Giunta, E. y Zamboni McCormick, K. (eds.) Teaching Italian American Literatura, Film and Popular Culture (pp. 70-78). The Modern Language Association of America.

Ponzanesi, S. (2004). Paradoxes of Postcolonial Culture. Contemporary Women Writers of the Indian and Afro-italian Diaspora. State University of New York.

Ponzanesi, S. y Merolla, D. (ed.) (2005). Migrant Cartographies: New Cultural and New Literary Spaces in Postcolonial Europe. Lexington Books. Rosendhal Thomsen, M. (2008). Mapping World Literature. International Canonization and Transnational Literatures. Continuum.

Russo Bullaro, G. y Benelli, E. (Ed.) (2014). Shifting and Shaping a National Identity: Transnational Writers and Pluriculturalism in Italy Today. Troubador

Sinopoli, F. y TAтti, S. (dir.) (2005). I confini della scrittura. Il dispatrio nei testi letterari. Cosmo Iannone Editore.

Sinopoli, F. (2012). Verso un concetto transnazionale delle scritture letterarie italiane. En Beniscelli, A., Marini, Q., Surdich, L. (Comp.). La letteratura degli italiani. Rotte, confini, passaggi (pp. 247-264). Città del Silenzio Edizioni.

Sinopoli, F. (2013). Postcoloniale italiano. Tra letteratura e storia. Novalogos Sinopoli, F. (2014). Interculturalità e transnazionalità della letteratura: questioni di critica e studi di casi. Bulzoni.

Tirabassi, M. (Comp.) (2005). Itinera. Paradigmi delle migrazioni italiane. Edizioni della Fondazione Giovanni Agnelli

Tirabassi, M. y Audenino, P. (2008). Migrazioni italiane: dall'ancien régime a oggi. $\mathrm{B}$. Mondadori.

Vertovec, S. (2009). Transnationalism. Routledge.

\section{Sinopoli, Franca}

«Reflexiones sobre la dimensión transnacional de la literatura contemporánea en Italia y en Europa». El hilo de la fábula. Revista anual del Centro de Estudios 